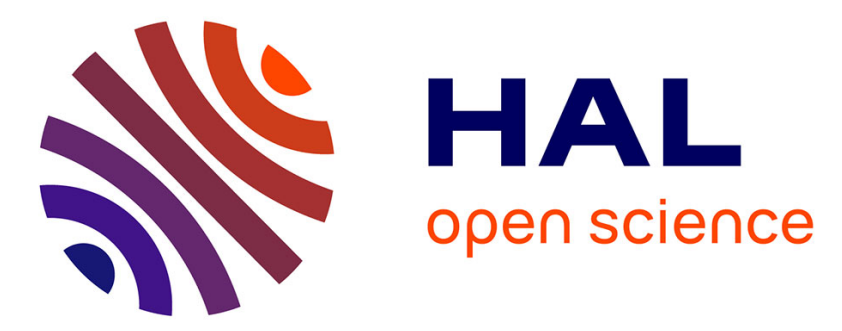

\title{
Les territoires pluriels des intermédiaires du marché du travail
}

\author{
Jean-Marie Pillon, Delphine Remillon, Carole Tuchszirer
}

\section{To cite this version:}

Jean-Marie Pillon, Delphine Remillon, Carole Tuchszirer. Les territoires pluriels des intermédiaires du marché du travail. La Revue de l'IRES, 2019, vol. 98 (n² 2), pp. 29-57. 10.3917/rdli.098.0029 . hal-02273201

\section{HAL Id: hal-02273201 \\ https://hal.science/hal-02273201}

Submitted on 28 Aug 2019

HAL is a multi-disciplinary open access archive for the deposit and dissemination of scientific research documents, whether they are published or not. The documents may come from teaching and research institutions in France or abroad, or from public or private research centers.
L'archive ouverte pluridisciplinaire HAL, est destinée au dépôt et à la diffusion de documents scientifiques de niveau recherche, publiés ou non, émanant des établissements d'enseignement et de recherche français ou étrangers, des laboratoires publics ou privés. 


\title{
Les territoires pluriels des intermédiaires du marché du travail
}

\author{
Jean-Marie PILLON, Delphine REMILLON et Carole TUCHSZIRER ${ }^{1}$
}

Cet article porte un nouveau regard sur la place des territoires dans les politiques de l'emploi en s'intéressant à l'activité des intermédiaires locaux du marché du travail. Il questionne la relation que ces intermédiaires instaurent avec les territoires en analysant les rapports qu'entretiennent deux types d'acteurs majeurs, les structures municipales et les agences locales de Pôle emploi. En mobilisant les données d'une enquête qualitative sur deux terrains, nous montrons que le territoire est à la fois un lieu d'application des politiques de l'emploi par des acteurs situés à différentes échelles (macro, méso, micro), porteurs de différentes conceptions du territoire (économique, politique, administrative), et un espace, géographiquement situé, doté d'une dynamique économique et sociale propre qui détermine en partie l'échelle d'intervention la plus pertinente. Cette diversité d'approches pose la question des instances de coordination à même d'imbriquer la multiplicité des manières de se saisir de la variable territoriale. Sur chacun de nos terrains on observe une convergence des interventions des intermédiaires, mais à une échelle géographique distincte, reflet des spécificités locales. Cette synchronisation des interventions se trouve toutefois bousculée par les réformes des politiques de l'emploi qui attisent les concurrences locales.

1. Maître de conférences à l'Université Paris Dauphine, Irisso-UMR 7170, affilié au Centre d'études de l'emploi et du travail (CEET), jean-marie.pillon@dauphine.psl.eu; Chargée de recherche à I'Institut national d'études démographiques (Ined), affiliée au CEET, accueillie au LiRIS, université de Rennes, delphine.remillon@ined.fr ; chercheure au Lise, Cnam et affiliée au CEET, carole.tuchszirer@lecnam.net. Merci à Solveig Grimault et aux participants du séminaire de l'Ires pour leurs commentaires et suggestions. Cet article s'appuie sur une enquête cofinancée par Pôle emploi et le CEET, menée avec nos collègues Yannick Fondeur, Anne Fretel et Claire Vivés, qui ont largement contribué à l'émergence de ces réflexions. Un grand merci enfin aux interviewés, les responsables des structures, d'avoir pris le temps de nous recevoir et de répondre à nos questions. 
Depuis plus de trois décennies, les territoires ont constitué une nouvelle catégorie d'action des politiques publiques (Duran, Thoenig, 1996 ; Poupeau, 2013) notamment dans le champ de l'emploi. Cet ancrage aux territoires a fait émerger une pluralité d'acteurs publics et privés qui participent au déploiement de ces politiques au point que la notion d'action publique est venue se substituer à celle de politique publique pour souligner la moindre place prise par l'État central dans un domaine de compétence de plus en plus partagé. Sur le terrain, la réalité de ce partage est plus nuancée et le rapport de l'État aux territoires plus ambigu (Epstein, 2015 ; Marcou, 2012 ; Bezes, Le Lidec, 2010). La littérature académique et les rapports publics montrent que le positionnement de l'État oscille entre deux postures et conceptions du territoire : l'une, un territoire de projection où l'État reste le pilote de la politique de l'emploi dans une logique descendante qui ne voit dans le territoire qu'un simple espace administratif de déploiement de son action ; l'autre, un territoire de projet, inscrit la politique de l'emploi dans une optique de développement local, à la main des acteurs locaux avec le soutien de l'État pour favoriser le déploiement de telles initiatives (Berthet et al., 2002a ; Berhuet, 2012 ; Chi Doan, 2015 ; Simha, 2013 ; Borel, Ferras, Le Coz, Ville, 2013). Ces hésitations quant à la prise en compte du fait territorial dans les politiques de l'emploi invitent à questionner les orientations de ces dernières et leurs effets globaux. C'est l'objet de cet article en s'intéressant aux acteurs centraux dans la mise en œuvre de ces politiques : les intermédiaires du marché du travail.

Par intermédiaires du marché du travail, nous entendons l'ensemble des organismes mobilisés par des programmes de retour à l'emploi des chômeurs ou de placement de candidats en entreprise et qui font donc fonction d'intermédiaires entre l'offre et la demande de travail. Nous mobilisons les données collectées dans le cadre d'une enquête qualitative réalisée entre 2014 et 2016 sur deux bassins d'emploi (encadré) pour analyser la relation que les intermédiaires instaurent avec les territoires. Il est apparu lors de l'enquête qu'il était difficile, pour les intermédiaires, de s'appuyer sur une définition stabilisée du territoire. D'un côté, l'espace local est l'arène d'un jeu d'acteurs complexe, acteurs situés à différents niveaux et tous porteurs d'une représentation particulière de leur mission sur le marché du travail et de leur légitimité dans la conduite des politiques de l'emploi (Berthet, 2008:90). Mais de l'autre, le territoire est également un espace, géographiquement situé, doté de sa propre dynamique économique et sociale à travers le profil des entreprises qui y sont implantées et des actifs qui y résident. Ce sont avec ces deux réalités du territoire que les acteurs locaux jonglent pour ajuster leur échelle d'intervention aux profils des publics à accompagner et aux réalités économiques des territoires. D’où les deux questions auxquelles nous cherchons à répondre dans cet article : À quelle 
échelle et selon quelle conception du territoire chaque intermédiaire du marché du travail déploie-t-il son action localement ? Comment s'organise la coordination, au niveau local, d'acteurs enjoints par différents canaux de commandement à mettre en œuvre des politiques portant sur un même champ ? Il s'agit là d'une question d'autant plus saillante que la composante « descendante » des territoires, jamais univoque, tend à construire des périmètres d'ampleur variable, selon des logiques d'action différentes et des temporalités désynchronisées. Nous avons aussi cherché à analyser la façon dont les intermédiaires locaux du marché du travail se débrouillent avec les évolutions relatives à la prise en compte de la variable territoriale dans les politiques de l'emploi mais aussi la manière dont les réformes successives bousculent leur périmètre d'action et les relations entre eux. Comment varient les procédures d'ajustement des politiques nationales au niveau local, par qui sont-elles organisées et en fonction de quels intérêts?

Pour répondre à ces questions, nous prenons plus particulièrement pour révélateur les relations de deux intermédiaires jouant un rôle majeur dans l'animation des politiques d'emploi au niveau local - les agences de Pôle emploi et les structures liées aux élus locaux au niveau (inter)communal. En effet, si l'Agence nationale pour l'emploi (ANPE) a traditionnellement occupé une place prépondérante sur les marchés locaux du travail (Canceill, Pignoni, 2000 ; Legay, Monchatre, 2000), la création de Pôle emploi a encore accru la revendication de l'opérateur national de jouer un rôle actif dans le pilotage local des politiques de l'emploi. D'un autre côté, des acteurs (inter)communaux et élus locaux - officiellement dépourvus de compétence en matière d'emploi - peuvent jouer un rôle central sur le territoire, par le biais des maisons de l'emploi ou d'autres structures ad hoc. Nous analysons donc plus particulièrement l'activité de ces structures dans leurs rapports avec les politiques de l'emploi décidées au niveau national, afin de rendre compte des dynamiques locales différenciées observées sur nos deux terrains.

Nous montrons que, localement, les différents intermédiaires interviennent à des échelles géographiques plus ou moins larges - d'échelles très étendues, voire a-territorialisées, à des échelles plus restreintes comme le bassin d'emploi, la commune ou le quartier, en lien avec les dynamiques économiques, le type de public accueilli et leur modèle d'intermédiation. Ils sont également porteurs de conceptions différentes du territoire. En toile de fond, nous montrons comment les multiples réformes de la politique de l'emploi territoriale et hésitations du rapport de l'État au local ont pu compliquer la tâche des acteurs locaux et attisé la concurrence entre eux, notamment entre les maisons de l'emploi, structures aux mains des élus locaux, et Pôle emploi. Dans un contexte d'incertitude institutionnelle, chacun tente en effet d'imposer sa vision du territoire, politique et économique 
d'un côté, administratif de l'autre. Du fait de la désynchronisation de ces échelles d'intervention et conceptions du territoire, les acteurs se trouvent confrontés à la question des instances permettant de coordonner l'intervention des intermédiaires au niveau local. Celles-ci prennent des formes variées et l'on observe une convergence des intermédiaires à une échelle différente sur nos deux terrains. Ce qui demeure néanmoins commun aux deux terrains est le fait que cette synchronisation des interventions est bousculée par les réformes, mais que malgré celles-ci les acteurs tentent toujours de se mobiliser, et parfois se coordonnent avec succès comme c'est le cas sur un des deux territoires observés, pour ajuster leur modèle d'intermédiation à la variété des publics accompagnés.

\section{Encadré}

\section{Le dispositif d'enquête}

Cet article fait suite à un projet de recherche collectif co-financé par la direction des études de Pôle emploi. L'enquête a été menée par une équipe de chercheur.es du Centre d'études de l'emploi et du travail (CEET). Cet article reprend et développe certains éléments du rapport final (Fretel et al., 2016). Dans le cadre de ce projet, une soixantaine d'entretiens ont été menés au sein de deux territoires, un territoire rurbain limitrophe d'une grande agglomération régionale (que nous nommerons ci-après Rurbain platlog) et une commune située en Île-de-France (que nous nommerons Banlieue IdF). Sur les deux terrains, ont été enquêtés des acteurs de l'emploi privés - entreprises de travail temporaires (ETT), opérateurs privés de placement (OPP), organismes de formation - ; les acteurs du service public de l'emploi (SPE) - Pôle emploi et ses agences spécialisées, missions locales pour les jeunes, Cap emploi pour les travailleurs handicapés - et leurs tutelles - notamment la Direction régionale des entreprises, de la concurrence, de la consommation, du travail et de l'emploi, Direccte - ; d'autres structures publiques ou associatives - association pour l'emploi des cadres (Apec), structures d'accompagnement des bénéficiaires du revenu de solidarité active (RSA), structures municipales, dont les maisons de l'emploi et les plans locaux d'insertion par l'économique (Plie), des structures d'insertion par l'activité économique (IAE), etc. L'objectif était de réaliser une cartographie des intermédiaires du marché du travail présents sur les territoires en analysant la diversité des modèles économiques et d'intermédiation ainsi que les relations entre les différents acteurs. Les entretiens ont été menés le plus souvent auprès de responsables de structures ou d'équipes. Le guide d'entretien a été adapté aux différents types d'acteurs mais comprenait systématiquement des questions sur le modèle économique des structures, les modes d'intermédiation, leur positionnement par rapport à l'offre et la demande, la territorialité de leur activité, leurs relations avec les autres intermédiaires. 


\section{Du territoire prescrit au territoire réel : des intermédiaires à la recherche de la bonne échelle d'intervention}

Cette première partie analyse le rapport au territoire des différents intermédiaires du marché du travail. Par rapport au territoire, précisons que nous entendons à la fois la conception du local qu'ils portent - vision ascendante ou descendante, politique et économique ou administrative du territoire - et leur périmètre géographique d'intervention. Après avoir proposé une formalisation de la pluralité des échelles territoriales auxquelles se déploient les actions des intermédiaires du marché du travail, nous analysons plus spécifiquement comment, sur deux territoires, les acteurs municipaux et Pôle emploi jouent leur partition. Nous montrons ainsi que, dotés de marges relatives dans l'interprétation des réformes, les intermédiaires du marché du travail mobilisent chacun une vision du territoire qui leur est propre. La juxtaposition des logiques descendantes et ascendantes fait du territoire un espace de "territorialisation concurrentielle » (Bartoli, Mériaux, 2011) entre les représentants de l'État (préfet, souspréfet, Direccte), les collectivités territoriales (région, département, communauté d'agglomération, commune) et les multiples opérateurs intervenant en leur nom (Pôle emploi, mission locale, Plie, maisons de l'emploi, associations, etc.).

\section{I.1. Des intermédiaires du marché du travail qui déploient leur action sur trois échelles territoriales}

Pour rendre compte des configurations entrevues au cours de notre enquête, nous présentons le contexte socioéconomique des deux territoires enquêtés, les acteurs en présence et distinguons trois échelles d'intervention typiques des intermédiaires du marché du travail : les niveaux macro (national, régional ou départemental), méso (bassin d'emploi) et micro (la commune voire le quartier).

L'enquête a porté sur deux territoires dénommés par la suite « Rurbain platlog » et «Banlieue IdF ». Rurbain platlog se situe dans le sud-est de la France, au nord d'un département peuplé d'1,2 million d'habitants et administré par une préfecture relativement éloignée. Le bassin économique du chef-lieu de département, spécialisé dans l'ingénierie innovante, est largement déconnecté de l'activité de Rurbain platlog. Ce dernier est plutôt rattaché à l'agglomération la plus proche, peuplée d'1,5 million d'habitants, chef-lieu de région mais située dans le département limitrophe. La cohérence du territoire découle en partie de son histoire récente. En 1968, l'État décide de créer une ville nouvelle sur ce territoire, dans le cadre de la politique dite des «métropoles d'équilibre ». À la suite de cette décision, des aménagements importants sont effectués à la fois sur le plan du bâti résidentiel (construction de grands ensembles livrés dès 1973) et économique 
(création de la zone d'activité centrale de notre étude en 1971). La ville nouvelle regroupe alors cinq communes rurales de petite taille. Au cours des années 2000, la gestion de la ville nouvelle s'étiole pour prendre fin en 2005. Elle est progressivement remplacée par une communauté d'agglomération qui regroupe 21 communes et près de 100000 habitants. Ce territoire se caractérise, du point de vue des intermédiaires, par le très grand nombre d'entreprises de travail temporaire (ET'T). Cette densité s'explique par la présence de nombreuses plateformes logistiques, qui concentrent l'essentiel des emplois de la zone, partagés avec l'industrie. Du côté des intermédiaires publics, on trouve sur la zone deux agences locales et une agence spécialisée de Pôle emploi, un réseau de missions locales constitué de trois antennes, et une agence Cap emploi. À ceux-là s'ajoutent plusieurs structures de l'insertion par l'activité économique (IAE). Il n'existe pas de plan local d'insertion par l'économique (Plie) ni de maison de l'emploi (MDE) sur le territoire enquêté mais plusieurs municipalités proposent des «structures de l'emploi municipales » occupant un à deux agents et destinées à répondre aux demandes des administrés.

Le second territoire enquêté est situé en Île-de-France, dans un département de la première couronne, nous l'appellerons par la suite Banlieue IdF. Il s'agit d'une commune qui compte plus de 80000 habitants. C'est un territoire dynamique économiquement, avec encore quelques grandes entreprises industrielles (même si l'une d'entre elles a quitté le territoire peu de temps avant l'enquête). La proximité de Paris et de grands axes et infrastructures de transports (trois autoroutes, un aéroport) ainsi que le prix peu élevé du foncier en font un territoire attractif pour les entreprises, notamment celles des secteurs du fret et de la logistique. Le bassin d'emploi est plus large que le strict territoire communal. Les habitants ne travaillent d'ailleurs pas majoritairement dans leur commune de résidence : c'est le cas de seulement $25 \%$ d'entre eux, tandis que $25 \%$ travaillent dans une autre commune du département et $50 \%$ dans un autre département - essentiellement Paris et la zone aéroportuaire. Il existe cependant un écart important entre le niveau de développement économique du territoire et le bénéfice que les habitants en retirent. La population est plus jeune (45\% ont moins de 30 ans) et moins diplômée que la moyenne (31 \% n’ont aucun diplôme). Au niveau résidentiel et sociodémographique, le peuplement de la commune est très contrasté, avec des quartiers pavillonnaires aisés en centre-ville, où les plus de 60 ans sont surreprésentés, et des quartiers plus excentrés et éloignés des transports publics où se concentrent les grands ensembles et où les jeunes sont surreprésentés (quartiers «Contrats urbains de cohésion sociale », CUCS et «zone urbaine sensible », ZUS, zone franche urbaine). Le taux de chômage est élevé (18,6 \% en 2012), avec des demandeurs d'emploi 
ayant un niveau de formation faible et beaucoup rencontrent des difficultés avec les savoirs de base (primo-arrivants mais aussi immigrés de plus longue date). Les emplois auxquels peuvent espérer accéder ces demandeurs d'emploi sont des emplois de proximité et peu qualifiés (nettoyage, magasinage, conduite/livraison, manutention, sécurité, vente, services à la personne) donc sur des niveaux de qualification très éloignés de ceux proposés par les deux grosses entreprises industrielles du territoire, qui embauchent peu sur place. Le tissu dense d'activités et d'entreprises d'un côté, et les difficultés d'emploi des résidents de l'autre, font de cette localité une zone d'action privilégiée pour les intermédiaires de l'emploi et ceux-ci sont effectivement très nombreux. Le territoire comprend une MDE, de nombreux acteurs du SPE (une agence Pôle emploi locale et une agence spécialisée, une mission locale, un Cap emploi, une structure pour les allocataires du revenu de solidarité active - RSA), huit structures d'insertion par l'activité économique, de nombreuses associations qui s'occupent des problématiques sociales mais font parfois aussi de l'insertion économique, des réseaux de soutien aux créateurs d'entreprise et des opérateurs privés (agences d'intérim, organismes de formation, organismes privés de placement, cabinet de reclassement).

Comment rendre compte du territoire d'intervention de ces différents intermédiaires de façon comparée sur nos deux terrains ? Pour répondre à cette question, nous avons analysé quel était le vivier de recrutement en matière de demandeurs d'emploi pour chacune des structures ainsi que sa zone de prospection des entreprises. Cette analyse nous a conduits à distinguer, de façon schématique, trois niveaux d'intervention.

\section{Macro : des acteurs au périmètre national, régional ou départemental, presque a-territoriaux}

Un certain nombre d'acteurs ont un ancrage territorial particulièrement faible. Leur échelle d'intervention est très étendue, voire a-territoriale. Outre les intermédiaires en ligne (Fondeur, 2016) on trouve surtout dans cette catégorie les intermédiaires qui se destinent aux cadres comme l'Association pour l'emploi des cadres (Apec). Cette structure privilégie une implantation centralisée (dans un centre régional ou à travers une interface dématérialisée) en concordance avec le périmètre de recherche d'emploi de son public, les cadres, qui se situe à une échelle régionale, nationale, voire internationale. De fait, on ne trouve pas d'agence Apec, dans aucune des deux localités étudiées. On trouve également à cette échelle des intermédiaires dont le public n'est pas suffisamment dense pour justifier d'un guichet local - c'est le cas des Cap emploi qui ont une emprise départementale, en cohérence avec leurs financements par l'Association de gestion du fonds pour l'insertion professionnelle des handicapés (Agefiph) et les 
conseils départementaux. D’autres organismes enfin (opérateurs privés de placement, organismes de formation) sont aujourd'hui incités à la concentration et partant, poussés à étendre leur périmètre d'intervention pour candidater aux appels d'offres désormais centralisés au niveau régional, au détriment de leur ancrage local.

S’il peut paraître étrange de ranger des structures d'envergure régionale ou départementale dans la case «macro », c'est pour signifier que les administrations ou organismes qui se déploient à cette échelle ont en fait des liens assez lâches avec les territoires étudiés et, surtout, avec les demandeurs d'emploi qui s'y trouvent. L'éloignement géographique rend tout déplacement de cet ordre inhabituel et complexe pour la plupart des chômeurs et des structures qui, comme on le verra, se situent à une échelle d'intervention bien plus restreinte. Le fait que certains intermédiaires ne disposent pas ou peu d'ancrage local ne signifie pas pour autant que les demandeurs d'emploi et les employeurs des territoires enquêtés soient totalement exclus de leurs services - à titre d'exemple, les demandeurs d'emploi cadres du territoire 2 peuvent se rendre à l'agence Apec dont ils dépendent, dont le périmètre d'action couvre trois départements franciliens et dont les locaux sont situés à l'autre bout du département. Cela signifie en revanche que les opportunités d'embauche auxquelles ces structures donnent accès ont peu d'ancrage local. Ces organismes peuvent en retour éprouver des difficultés à capter des candidats venant de zones spécifiques - c'est le cas de l'Apec avec les jeunes diplômés du territoire 2, alors qu'elle dispose de financements spécifiques pour eux - et à nouer des relations avec les acteurs locaux de l'emploi.

\section{Méso : des acteurs intervenant à l'échelle du bassin d'emploi}

D’autres intermédiaires enquêtés ont un ancrage territorial de périmètre moyen, relatif au bassin d'emploi. Il s'agit de structures qui participent à la circulation de la main-d'œuvre au sein d'un tissu productif relativement cohérent, aux yeux des acteurs. Cette échelle rappelle les zones d'emploi de l'Institut national de la statistique et des études économiques (Insee), définies comme un espace géographique à l'intérieur duquel la plupart des actifs résident et travaillent, et dans lequel les établissements peuvent trouver l'essentiel de la main-d'œuvre nécessaire pour occuper les emplois offerts. Dit autrement, ces intermédiaires ont un ancrage qui est déterminé par le tissu économique local. On retrouve dans cette catégorie les structures qui suivent des populations dont la profession ou le métier est bien identifié et dont les qualifications sont qualitativement ajustées aux opportunités disponibles sur le marché du travail local. Il s'agit d'une échelle qui découle des politiques de main-d'œuvre des employeurs et à laquelle il est possible de vivre de l'intermédiation, comme le font les agences d'intérim. 
Employeurs comme candidats souhaitent pour la plupart restreindre leurs recherches à l'échelle du bassin pour minimiser les risques et les coûts associés à une embauche trop éloignée du lieu de résidence.

En théorie, les agences locales de Pôle emploi sont destinées à intervenir à ce niveau. Leur mission officielle et surtout les outils dont elles disposent pour la mettre en ouvre sont en effet fortement structurés par l'objectif de rapprocher offre et demande à l'échelle d'un bassin d'emploi. Comme nous le montrons plus loin, le quotidien de cet établissement, et notamment les caractéristiques de son public, l'éloigne néanmoins bien souvent de ce périmètre d'intervention. D'où le déploiement de certains services d'accompagnement à une échelle plus micro, souvent par le biais d'une délégation d'accompagnement à des structures qui ont une connaissance plus fine du territoire (la mission locale pour les jeunes par exemple).

\section{Micro : des acteurs intervenant à l'échelle de la commune voire du quartier}

Une partie des intermédiaires, enfin, situe son intervention au niveau le plus local, indépendamment du tissu économique, et sur la base du lieu de résidence des candidats. Il s'agit d'intermédiaires qui ont d'abord pour vocation d'accueillir les demandeurs d'emploi avant d'entreprendre de les rapprocher de l'offre, et d'effectuer un suivi social, comme les structures municipales ou les missions locales. Nous rangeons également dans cette catégorie les acteurs chargés d'accompagner les allocataires du RSA. Bien que rattachés officiellement - directement ou indirectement - au conseil départemental, ceux-ci sont le plus souvent localisés voire communalisés. On remarque, comme Berthet et alii (2002b), que les acteurs qui mettent en œuvre les modèles d'accompagnement les plus individualisés et renforcés sont également les plus territorialisés. La localisation géographique de ces intermédiaires locaux est cruciale en ce qu'elle détermine leur capacité à atteindre des franges de la population qui ne sont pas immédiatement connectées au marché du travail et au service public de l'emploi. Ce positionnement est pensé à un niveau géographique très fin, parfois infracommunal à l'échelle du quartier. Nous plaçons aussi dans cette catégorie les entreprises d'insertion par l'activité économique, et plus encore les chantiers d'insertion, qui sont bien souvent structurés sur une base locale voire communale. Ces organismes assurent des activités que l'on retrouve dans la plupart des communes : bâtiment, services à la personne, entretien d'espaces verts, recyclage, etc. Nécessitant peu de qualifications certifiées, ce sont là des activités qui permettent d'envisager un retour à l'emploi compatible avec une mobilité à l'échelle de la commune.

La maison de l'emploi que nous avons étudiée sur le territoire Banlieue IdF relève de cette échelle d'intervention. Institutionnellement et 
financièrement, elle se trouve fortement rattachée à la commune et les spécificités socioéconomiques de celle-ci conduisent la maison de l'emploi à concentrer son activité sur les publics des quartiers les plus en difficulté du territoire. Néanmoins, certaines autres de ses missions, comme celles relatives au développement économique du territoire, auraient plus de pertinence à une échelle méso comme nous le montrerons ensuite.

Comme en atteste cette représentation quelque peu modélisée du territoire d'intervention des intermédiaires de l'emploi, ces derniers sont ainsi aux prises avec des modèles d'action divers (Marchal, Bureau, 2009) et des échelles d'intervention plurielles. Cette organisation pourrait paraître rationnelle a priori, ces différentes échelles d'intervention étant le produit d'un ajustement aux périmètres réglementaires, aux problématiques spécifiques de l'activité économique locale et aux contraintes de mobilité des demandeurs d'emploi suivis. Cependant, dans les faits les choses ne sont donc pas si claires que cela. Certaines structures peuvent déployer leurs actions à plusieurs échelles territoriales en fonction de leurs différentes missions. C'est le cas des deux principaux intermédiaires locaux que nous avons eu l'occasion de rencontrer : les MDE et Pôle emploi. Dans le cas de Pôle emploi, on constate une relative contradiction entre ses outils, sa logique administrative et descendante qui le poussent vers le niveau méso tandis que le public accompagné, c'est-à-dire celui qui bénéficie le moins des opportunités d'emplois, favorise une focalisation sur le micro. Dans le cas des maisons de l'emploi, leurs missions de développement économique n'ont de sens qu'au niveau méso. Mais dans le même temps, certaines logiques politiques consistant à " rendre service à la population » ou à défendre auprès d'elle un «bilan » la tirent vers le niveau micro. En outre, des réformes politiques en arrière-plan peuvent venir bousculer des logiques d'action territoriales et périmètres d'intervention bien installés. À cet égard, l'exemple des relations entre Pôle emploi et les maisons de l'emploi est là encore emblématique. C'est pourquoi nous allons à présent nous attarder sur le rapport au territoire de ces deux intermédiaires.

\section{I.2. Entre développement économique et accompagnement des publics : des maisons de l'emploi au cœur de controverses territoriales}

L'épisode qui s'ouvre en 2005 à l'occasion de l'adoption de la loi de cohésion sociale dite « loi Borloo » est représentatif des signaux contradictoires que l'État central adresse aux acteurs locaux, intermédiaires du marché du travail, élus locaux et échelons déconcentrés du service public de l'emploi. Les maisons de l'emploi ont pour vocation à l'origine d'accueillir, sous le même toit, tous les acteurs locaux de l'emploi. Les porteurs de projet sont les collectivités territoriales, donc des élus locaux, auxquels doivent 
se joindre obligatoirement l'État, l'ANPE et l'Assedic ${ }^{2}$ mais également, si les porteurs le souhaitent, les conseils régional et départemental, les communes et intercommunalités, l'agence pour la formation professionnelle des adultes (Afpa), les organismes consulaires, les partenaires sociaux, l'Apec. La loi Borloo assigne à ces maisons de l'emploi des missions qui recoupent en partie celles de l'ANPE, notamment celle de l'accompagnement des chômeurs (Balzani et al., 2008).

À cette première difficulté d'ordre institutionnel vient s'ajouter l'ambivalence des missions assignées à ces maisons de l'emploi quant à leur ancrage au territoire. Dans l'exposé des motifs de la loi qui justifie leur création, trois conceptions du territoire se juxtaposent plus qu'elles ne se recoupent, rendant les objectifs difficilement conciliables. Les maisons de l'emploi doivent :

- « associer les collectivités territoriales, fédérer l'action des partenaires publics et privés et ancrer le SPE dans les territoires »;

- « assurer la convergence des politiques publiques de l'emploi adaptées au développement économique et social du territoire »;

- « assurer la coopération entre partenaires autour d'un projet de territoire construit à partir d'un diagnostic, d'objectifs, d'un plan d'action, d'une programmation et d'une évaluation partagés ».

Les deux premières missions attribuées aux maisons de l'emploi renvoient à une approche du fait territorial comme lieu de mise en œuvre de la politique nationale dans une logique de déconcentration : la maison de l'emploi incarne le service public de l'emploi au niveau local, constitue une caisse de résonance des politiques publiques de l'emploi en tâchant de les ajuster aux spécificités locales. L’originalité du projet consiste à faire coexister au sein d'un même lieu cette approche « descendante » avec une approche plus « ascendante » qui peut paraître contradictoire. Le troisième objectif se trouve en effet à contrecourant des deux précédents puisqu'il fait des maisons de l'emploi l'outil du territoire à partir duquel doit se construire un projet visant son développement, sans référence aucune au SPE. Il s'avèrera dès lors difficile de faire cohabiter dans ces maisons de l'emploi ces formes de mobilisation contradictoires du territoire (Berhuet, 2012).

En outre, dès 2007, l'arrivée d'un nouvel exécutif et la création, en 2008, de Pôle emploi, qui institutionnalise le principe du guichet unique intégré, correspondent à une période de recentralisation du SPE et de marginalisation progressive des maisons de l'emploi (Berhuet, Tuchszirer, 2015).

2. L'Agence nationale pour l'emploi (ANPE) avait pour mission d'accompagner les chômeurs dans leur recherche d'emploi et d'aider les entreprises à recruter. Les Assedic, associations pour l'emploi dans l'industrie et le commerce, avaient pour mission de percevoir les cotisations chômage et d'indemniser les demandeurs d'emploi. Ces deux organismes ont été fusionnés en 2008 (création de Pôle emploi). 
Leur cahier des charges est modifié et leurs missions sont progressivement réduites, jusqu'à leur retirer le droit d'accueillir du public et d'accompagner des chômeurs.

Ces allers et retours dans la répartition institutionnelle du travail sont sources de tensions sur le terrain, de concurrence entre les intermédiaires ainsi que l'observent ces directeurs d'une maison de l'emploi et d'une agence Pôle emploi :

«Dans le secteur associatif, on a tendance à pallier les manques. Là où il y a des interstices, on va y aller, parce qu'il y a un besoin (...) ; et du jour au lendemain, on va combler l'interstice et on va nous dire : "Non mais attendez, non, c'est la mission de Pôle emploi." (...) On est dans un contexte extrêmement peu sûr, à la fois d'un point de vue purement et strictement institutionnel, mais aussi financier, et du coup, ça génère des tensions sur les territoires » (directeur d'une maison de l'emploi).

«Moi, le souvenir que j'ai, c'est, l'ANPE, on était obligé de se battre bec et ongles pour ne pas que la maison de l'emploi devienne une structure concurrente. Nous, on est l'outil d'une politique nationale et finalement les maisons de l'emploi, les missions locales sont l'outil d'une politique locale » (directeur d'une agence locale Pôle emploi).

L'émergence de structures associatives se positionnant comme intermédiaires locaux du marché du travail prolonge l'investissement historique des communes en matière de secours aux populations vulnérables (Castel, 1995 ; de Barros, 2001). Celles-ci ont depuis longtemps développé des activités d'intermédiation. Leur rôle en la matière a eu tendance à se renforcer avec l'apparition d'un nouveau registre de légitimation des politiques publiques, celui de la proximité (Berthet, 2010). C'est bien le cas sur les deux territoires enquêtés, Banlieue IdF disposant d'une maison de l'emploi communale qui abrite la mission locale et un pôle insertion pour l'accompagnement des adultes, et Rurbain platlog de plusieurs structures d'emploi municipales. Le périmètre d'intervention de ces structures est celui de la commune, unité électorale envers laquelle les élus sont responsables. Le territoire a pour ces structures une légitimité, une dimension politique, contrairement à ce qu'il en est pour Pôle emploi : «M. X de Pôle emploi, il ne s'adresse pas à la population, enfin il ne fait pas de publicité directe. Nous, on a un rôle de vitrine » (élu local, Banlieue IdF). Il s'agit selon le dire des élus locaux d'agir pour le bien de la commune et de ses administrés, de rendre service, de faire du « sur mesure » par opposition au modèle de masse des acteurs du SPE et en premier lieu de Pôle emploi :

«Pôle emploi ne fait pas l'accueil de demandeurs d'emploi tel que pouvaient le faire des services locaux, dans la proximité, parce que eux [Pôle emploi] ils font du chiffre et du contrôle. Mais il n'y a pas de suivi, pas d'accompagnement et il n'y a pas le lien un peu social que pouvaient et que 
peuvent avoir certains services publics, un service de l'emploi local, porté par des collectivités. Ce n'est pas du tout le même objectif, eux [les élus locaux] ce qu'ils veulent c'est rendre service à la population » (responsable du développement économique, Banlieue IdF).

Ces services municipaux accompagnent un petit nombre de personnes, celles qui les sollicitent car elles ne trouvent pas leur compte ailleurs. C'était le cas par exemple du pôle insertion de banlieue IdF (avant qu'il ne soit fermé suite au changement de cahier des charges des maisons de l'emploi) : «On va accueillir des gens qui sont ASS ${ }^{3}$, qui sont des primo-arrivants, qui ont des problèmes avec la barrière de la langue... ». L’objectif visé est de s'adapter aux besoins des usagers, avec moins de pression en termes d'objectifs de placement que ne peuvent en avoir les acteurs du SPE.

Ces acteurs municipaux contribuent aussi à animer un marché local du travail et à lier entre elles les différentes structures en présence (Baron et al., 1995). Ils mènent des actions de mobilisation des employeurs locaux et tentent d'attirer de nouvelles entreprises sur le territoire, seule action à même selon eux de résoudre les problèmes d'emploi, comme l'explique cet élu : «S'il n’y a pas d'emploi, vous pouvez toujours essayer de faire ce que vous voulez, il ne se passera rien pour les publics ». Les communes s'appuient pour ce faire sur le fait d'être en première ligne pour ce qui concerne l'implantation des entreprises : recherche de locaux disponibles, dépôt du permis de construire, adaptation de la voirie, etc. Les structures municipales rencontrées combinent ainsi souvent les deux missions - accueil de chômeurs et développement économique local, actions qu'elles jugent étroitement liées. En la matière, les élus peuvent porter une vision assez limitée du développement économique local, fondée sur le développement d'espaces et de zones (d'activité, commerciale, etc.). L'articulation entre les qualifications recherchées par les sociétés amenées à investir ces zones et les demandeurs d'emploi des communes qui les accueillent demeure secondaire et, surtout, peu planifiée. Pourtant, les mètres carrés ne créent pas automatiquement de l'emploi (Crague, 2018).

$\mathrm{Au}$ regard de la typologie des échelles d'intervention que nous proposions plus haut, les missions de développement économique ne sont pas positionnées officiellement à un échelon spécifique. L'apparition des Direccte dans le cadre de la réforme de l'administration territoriale de l'État (Réate) et l'inscription très claire des directions régionales de l'industrie, de la recherche et de l'environnement (Drire) au sein de ces nouvelles directions interministérielles plaide pour une prise en charge de cette mission à une échelle macro ou méso. Les acteurs municipaux ne sont toutefois pas exclus de ces opérations, à condition, disent-ils, de construire des

3. Allocation de solidarité spécifique. 
alliances à un niveau méso : "Si on veut élaborer une stratégie de développement économique, on ne peut pas le faire à l'échelle d'une ville » (responsable du développement économique, Banlieue IdF). Le rôle de la MDE pour coordonner ces différentes actions est d'ailleurs inscrit dans la loi de $2008{ }^{4}$. C'est même la principale mission qui leur reste depuis la modification du cahier des charges et l'interdiction d'accueil du public. À l'inverse, du côté de l'accompagnement, l'échelle d'intervention des structures municipales est parfois encore plus restreinte que la commune. Ainsi, la mission locale de Banlieue IdF opère en théorie sur l'espace géographique communal. Mais dans les faits, son aire d'influence est plus réduite. En effet, elle s'est implantée dans un quartier difficile de la commune en jouant la carte de la proximité avec les jeunes qui y résident, exclus du marché du travail, et donc qui constituent son public cible. Ce faisant, elle s'est peu à peu coupée du reste de la commune et notamment des jeunes des autres quartiers, plus pavillonnaires, qui ne s'y rendent plus. De même, la gestion des clauses d'insertion ${ }^{5}$, qui est une des activités de ces structures, impose souvent de descendre à un niveau infra-communal, notamment pour les clauses relatives aux rénovations urbaines (Agence nationale pour la rénovation urbaine, Anru) qui imposent d'embaucher des résidents de certains quartiers ou rues, comme l'explique la facilitatrice des clauses d'insertion de Banlieue IdF : «On est obligé de mettre des candidats des quartiers fragiles quand il y a des marchés Anru ». La problématique pour les structures municipales est donc d'articuler ces différentes échelles d'intervention, des plus locales, parfois infra-communales du côté de l'accompagnement des publics, à des échelles plus larges, au moins intercommunales du côté des problématiques de développement économique.

\section{I.3. Pôle emploi, entre vision administrative du territoire et nécessité d'un accompagnement de proximité pour les demandeurs d'emploi les plus en difficulté}

L'action de Pôle emploi se décline à différentes échelles, en lien avec la pluralité de ses missions (Legay, Monchatre, 2000) et les qualifications et aires de mobilité variées des demandeurs d'emploi suivis. C'est un organisme d'ampleur nationale qui développe un certain nombre d'outils a-territorialisés (donc de niveau macro), notamment son site Internet qui relaie l'ensemble des offres d'emploi collectées. L'ANPE est néanmoins organisée dès l'origine en agences locales. Son ancrage apparaît ainsi

4. Article 3 de la loi du 13 février 2008 : «La maison de l'emploi concourt à la coordination des politiques publiques et du partenariat local des acteurs publics et privés qui agissent en faveur de l'emploi, de la formation, de l'insertion et du développement économique. »

5. Les clauses sociales d'insertion sont un dispositif juridique permettant d'intégrer des considérations liées à la lutte contre le chômage et les exclusions dans les appels d'offres publics. Elles favorisent de fait l'accès des structures d'insertion par l'activité économique à la commande publique. Il existe deux types de clauses, celles relatives à l'Anru et celles qui figurent dans des marchés publics concernant des territoires de droit commun. 
fortement lié aux bassins d'emploi sur lesquels les agences sont implantées. Selon les cas, le territoire d'intervention d'une agence Pôle emploi peut correspondre à tout ou partie du territoire d'une commune ou s'étendre sur un périmètre plus large. L'agence a la responsabilité de l'inscription et du suivi des demandeurs d'emploi habitant au sein de cette zone et gère les offres d'emploi des entreprises qui y sont implantées. Pour autant, les offres sont en théorie en libre accès en tout point du territoire et Pôle emploi étant une organisation nationale, ses directeurs doivent rendre des comptes sur l'efficacité de leur administration, mais ne sont pas redevables au niveau local.

Pôle emploi n'a pas une conception "politique » mais « administrative » du territoire selon la distinction de Bel (2007). Il ne s'agit pas d'une structure qui doit rendre des comptes aux électeurs et ne favorise, de ce fait, aucunement les résidents d'une commune en particulier, contrairement aux acteurs municipaux. L'objectif consiste à décliner au niveau local, dans une « logique descendante » (Berthet et al., 2002a), une politique nationale qui a pour objectif de placer des demandeurs d'emploi et de satisfaire les entreprises, quel que soit leur lieu de résidence. Cette logique heurte celle des acteurs municipaux et est source d'incompréhensions mutuelles, comme l'explique la facilitatrice de Banlieue IdF à propos de la recherche de candidats pour pourvoir les offres d'emploi liées aux clauses d'insertion :

«Les offres [liées aux marchés clausés], elles sont quand même réservées en priorité pour le public de Banlieue IdF. (...) Sauf que quand on dépose une offre à Pôle emploi, le problème, c'est qu'on nous envoie des profils illimités. On peut avoir du 93, on peut avoir du 78, du 94, du 91 et moi, je me suis vu déjà avoir une pile comme ça, donc, à chercher mes petits [habitants de la commune] et je n'en avais pas. Donc, là, c'est vrai qu'il y a encore des choses à réajuster avec Pôle emploi par rapport au profil. »

Il s'agit là d'une autre opposition entre Pôle emploi et les acteurs municipaux, celle relative à l'échelle d'intervention territoriale privilégiée : de par ce maillage du territoire par les agences locales (à l'échelle des bassins d'emploi) et les dispositifs de mise en relation des offres d'emploi et des demandeurs d'emploi, Pôle emploi semble s'inscrire principalement à une échelle d'intervention méso. En effet, Pôle emploi a pour vocation de faire circuler les populations au sein de chaque bassin d'emploi (ou entre différents bassins d'emploi). Mais cet ancrage méso ne correspond que faiblement à un public peu qualifié et, partant, peu mobile, public majoritaire à Pôle emploi depuis 30 ans. Les différents outils dont disposent les agences locales - la pratique de l'e-mailing ou les envois postaux groupés mais aussi le répertoire opérationnel des métiers et des emplois de 
Pôle emploi (Rome ${ }^{6}$ ) - semblent relativement inappropriés pour suivre des demandeurs d'emploi dont les horizons et les opportunités ne sont pas fondés sur la qualification ou le métier ni sur une potentielle mobilité géographique, mais sur le lieu de résidence et la polyvalence d'emplois alimentaires. Le modèle d'intermédiation dominant que Pôle emploi met en œuvre et les outils qu'il mobilise s'avèrent ainsi relativement désajustés avec son public le plus fragile. D'où les diverses tentatives de Pôle emploi pour s'inscrire à des échelles d'intervention plus fines, en développant des dispositifs d'accompagnement renforcés des demandeurs d'emploi aux aires de mobilité très restreintes, dans le cadre d'un partenariat étroit avec des structures sociales de proximité. C'est la logique par exemple de la modalité d'accompagnement désignée sous le terme d'« accompagnement global » qui s’appuie sur un partenariat avec les conseils départementaux et les structures d'insertion de proximité pour suivre les demandeurs d'emploi qui multiplient les handicaps dans leur recherche d'emploi.

Ainsi, ce sont deux visions du territoire distinctes que portent Pôle emploi et les structures municipales, un territoire simple unité de découpage administratif d'un côté et, de l'autre, un territoire unité d'action publique au niveau économique et social, doté d'institutions redevables politiquement. L'une ou l'autre de ces conceptions et échelles territoriales ont été favorisées par l'État central au gré des réformes, ce qui crée de l'incertitude institutionnelle et de la concurrence sur le terrain, notamment entre ces deux acteurs majeurs de l'emploi au niveau local que sont Pôle emploi et les structures municipales. Comment ces tensions se résolvent-elles pour mener à bien, localement, des actions communes sur l'emploi ? Par ailleurs, toutes ces structures sont confrontées, on l'a vu, à la difficile question de l'articulation des échelles d'intervention, notamment entre l'échelle méso - qui est l'échelle privilégiée de Pôle emploi et l'échelle du développement économique, aux mains des élus locaux - et l'échelle micro - l'aire de mobilité privilégiée des demandeurs d'emploi les plus fragiles, qui sont les premiers touchés par le chômage. Cela nécessite de la coordination entre les intermédiaires pour imbriquer ces différentes échelles et problématiques territoriales.

\section{Quelles instances pour réguler et coordonner les politiques de l'emploi territoriales?}

Combiner des échelles territoriales d'intervention variées et passer d'une échelle à l'autre constitue un enjeu fort pour les intermédiaires de l'emploi. Les acteurs locaux s'interrogent sur les instances susceptibles

6. Le Rome de Pôle emploi définit, pour chaque métier, ses conditions d'exercice, les activités et compétences de base et spécifiques et, dans la rubrique « mobilité professionnelle », les métiers accessibles facilement ou via une formation. 
d'ajuster ces périmètres d'intervention disjoints. À l'aune de l'enquête de terrain, on constate deux éléments. D’une part, les modalités d'organisation des relations entre les acteurs et l'échelle géographique à laquelle s'effectue cette coordination varient largement d'un territoire à un autre ; d'autre part, les acteurs font preuve d'attentes importantes en matière de leadership et celui-ci est revendiqué par des instances diverses, ce qui peut conduire à des situations de concurrence institutionnelle, celles-ci étant encore accentuées par le flou chronique des réformes nationales.

\section{II.1. Des variations locales des instances de coordination des politiques de l'emploi}

Les politiques de l'emploi sont mises en œuvre de manière différente sur les territoires car les instances de coordination varient.

\section{Un « chef d'orchestre » pour les acteurs locaux?}

Le premier type d'instance que l'on rencontre pourrait être qualifié de « chef d'orchestre ». Ce type de coordination est le fait d'acteurs qui ont la légitimité de parler à l'ensemble des intermédiaires pour réguler leur intervention. C'est le cas, en ce qui concerne Rurbain platlog, de l'agent de la Direccte qui occupe le poste d'animateur économique territorial. Chargé de rendre le territoire attractif pour des investisseurs, ce dernier use de cette casquette pour mettre en place une organisation lisible et efficace du versant entreprise des politiques de l'emploi, un SPE « rationnalisé et simplifié », et se positionne ainsi en tant que régulateur et coordinateur des intermédiaires publics de l'emploi. Sans disposer de prise hiérarchique directe sur eux, il parvient néanmoins à les faire dialoguer régulièrement :

« [M]oi je veux régulièrement avoir les quatre [directeurs de Pôle emploi] en face pour qu'on définisse comment on travaille sur ce territoire. J'ai la mission locale, j'ai Cap emploi, les techniciens de la Région, donc les $\mathrm{CTEF}^{7}$ et les directeurs de la formation continue, j'ai les délégués du préfet pour la politique de la Ville (...) et j’ai les collègues du Conseil départemental, donc des trois territoires, les trois responsables d'insertion. C'est un groupe technique orientation-emploi-formation, où l'on essaye, une fois par trimestre, de donner des éléments d'actualité sur les dispositifs... (...) On a un temps d'information, et ensuite on échange sur les projets du territoire et quels sont les projets sur lesquels on va ensemble » (chargé d'animation territoriale Direccte, Rurbain platlog).

Sa stratégie consiste à faire de Pôle emploi le chef de file opérationnel des politiques de l'emploi au niveau local, quitte à déléguer ensuite

7. Contrats territoriaux emploi formation. 
l’intermédiation aux autres intermédiaires plus spécialisés, dans le handicap, l'insertion ou les jeunes travailleurs :

« À un moment donné, quand on a une entreprise qui cherche à recruter, elle se dit : "Je m'adresse à qui ?” (...) Moi, je vais avec Pôle emploi dans l'entreprise, ensuite Pôle emploi travaille et diffuse l'offre auprès de l'ensemble des acteurs. C'est la commande pour que ce soit visible. [Sinon], l'entreprise en face nous dit : “Attendez monsieur, moi je n'y comprends plus rien, je fais quoi là-dedans ?” En plus, chacun va essayer de vendre son dispositif » (chargé d'animation territoriale Direccte, Rurbain platlog).

En retour, son rôle de coordinateur est accepté et reconnu par tous les intermédiaires, et en premier lieu par Pôle emploi. Parlant de ce salarié de la Direccte, un directeur d'agence déclare :

« [Les personnes que je n'aurais pas rencontrées sans lui, ce sont] les grandes entreprises et différents acteurs. Il a un respect des différents acteurs privés et publics, il est l'animateur territorial, c'est son poste d'ailleurs. Il est très fort à tous les niveaux : il connaît les entreprises, les partenaires institutionnels, il connaît tous les aspects de formation, les compétences rattachées. »

S'il parvient à jouer ce rôle sur ce territoire, cela tient sans doute en partie à sa trajectoire professionnelle (plus de 20 ans d'ancienneté sur le poste). Il est néanmoins parvenu à jouer avec sa fiche de poste jusqu'à entretenir une proximité physique avec le bureau du sous-préfet qui n'était pas prévue initialement. Il se trouve ainsi parfois amené à assurer la fonction de chargé de mission emploi de la sous-préfecture - une fonction qui n'est pas dans ses attributions - qui lui donne accès à des informations de première main sur les installations d'entreprises à venir. Il inscrit ainsi son action en matière d'emploi dans une logique de développement économique du territoire et non dans une logique administrative de contrôle du service public de l'emploi et incite Pôle emploi à sortir de cette vision administrative :

«Souvent, c'est un peu la difficulté de Pôle emploi, c'est qu'eux ils ont une vision : "J'ai des offres, du coup, je mets des gens en face." Moi j'essaye, en discutant avec les entreprises aussi, de connaître un peu mieux leur stratégie, pour voir, cette entreprise, où elle en est, comment elle va évoluer, et quelles sont aussi les perspectives. On a par exemple deux, trois entreprises en développement, j'ai dit à Pôle emploi : "Il faut très bien les servir, il fait avoir une offre de service +++ " (...), parce que derrière il y a des perspectives de développement et qu'à terme, on s’y retrouvera. »

Si cette dimension de développement économique fait effectivement partie des missions des Direccte, dont la création a traduit la volonté de l'État de conduire des politiques axées sur le renforcement du lien travail/ emploi/développement économique (Crague, 2018), cette empreinte forte 
de la Direccte pour lier les deux ne s'observe pas sur tous les territoires. Mais sur nos différents terrains, la Direccte s'efforce effectivement de «mettre de l'ordre » dans le SPE en évitant les doublons et en redimensionnant les mandats des différentes structures. C'est également le cas à Banlieue IdF mais dans une perspective plus administrative. Aux dires des responsables de la maison de l'emploi en effet, Direccte et Pôle emploi sont par exemple « main dans la main » pour défendre le service public de l'emploi et réduire le champ d'action de la maison de l'emploi. Toutefois, à Banlieue IdF, il n'existe pas d'instance «chef d'orchestre » dont le rôle serait reconnu par tous les intermédiaires et la concurrence entre Pôle emploi et la maison de l'emploi pour tenir ce rôle est observable dans ces propos du directeur de l'agence locale de Pôle emploi :

«On a voulu donner ce rôle-là aux maisons de l'emploi, ce rôle d'intégrateur en fait, sauf que... Si je puis me permettre, et là c'est une appréciation personnelle, ce rôle d'intégrateur est difficile. Donc à mon sens, ça fonctionne s'il y a un vrai partenariat fort entre la maison de l'emploi et Pôle emploi. Mais très honnêtement, j'ai participé à cette évaluation [des maisons de l'emploi], mais moi, je ne crois pas que les maisons de l'emploi soient un outil nécessaire.

- Ce rôle d'intégrateur pour vous, c'est Pôle emploi qui doit le jouer? (...)

- Je trouve que ça doit être Pôle emploi, mais en travaillant avec un partenariat fort avec les politiques locaux. »

\section{Les réunions de concertation}

En l'absence de «chef d'orchestre », on trouve d'autres instances cherchant à coordonner les acteurs des politiques de l'emploi, par exemple en ce qui concerne la répartition des bénéficiaires du RSA à accompagner, celle des emplois des marchés clausés, des places de formation. Ainsi, sur le territoire 2 , se tient chaque mois une réunion réunissant le projet de Ville RSA, le Pôle emploi et le service social afin « d'étudier les listes d'orientation [des bénéficiaires du RSA] du Conseil général pour confirmer ou infirmer, avec proposition d'un nouveau service référent, l'orientation faite par le Conseil général. »Ces instances prennent le plus souvent la forme de réunions, où différents intermédiaires sont représentés et au cours desquelles un organisme administratif supra-territorial (qui n'a pas de guichet au niveau local ou pas de guichet tout simplement) se rapproche des acteurs locaux pour distribuer des ressources. Ces différentes instances de régulation constituent des arènes importantes pour les acteurs locaux. Ils y trouvent en effet des débouchés pour leur public (formations, places protégées, etc.). De plus, ils élaborent dans ce type de réunions différents critères de sélection et de hiérarchisation des dossiers qui servent ensuite 
en interne à évaluer la proximité des profils de leur public avec différentes formes de sorties positives. Au sein de ces instances, le travail de coordination est le plus souvent pris en charge par des collectivités territoriales. L'avantage de ces collectivités est qu'elles sont proches du terrain. Mais a contrario, elles sont aussi plus inscrites dans des rapports de force et luttes locales. C'est ce que tend à illustrer le cas de la maison de l'emploi du territoire 2 où les élus locaux, qui siègent au conseil d'administration aux côtés de la Direccte, conservent une forte influence. En témoignent les remous que subit la structure en période électorale. Ce sont les élus par exemple qui ont le pouvoir de décider de poursuivre ou non les missions que l'État (la Direccte) ne finance plus.

\section{Les conventions de gestion}

Un troisième type de régulation de la politique locale de l'emploi passe par des conventions bilatérales entre opérateurs. La notion de partenariat est particulièrement problématique en matière d'intermédiation en ce que la notion recouvre en fait bien souvent des relations de pouvoir et de soustraitance plutôt que la construction commune d'un service, comme en témoigne cette déclaration d'un directeur d'une agence Pôle emploi, pour qui le partenariat permet d' « étendre [s]a zone d'influence. »

Certaines relations bilatérales semblent néanmoins déboucher sur un bénéfice commun lorsqu'elles permettent de répartir les demandeurs d'emploi en fonction de l'échelle qui leur convient le mieux. Les liens tissés par Pôle emploi avec d'autres organismes reflètent cette fonction d'ajustement des échelles d'intervention. Après une période de repli sur soi, où les directeurs disent s'être consacrés en interne à absorber le choc de la fusion ANPE/Assedic, les lignes stratégiques édictées depuis 2012 (Pôle emploi 2015 et 2020) leur ont accordé des marges de manœuvre supplémentaires pour développer des partenariats locaux :

«Par rapport au volet partenarial, ça nous a donné des marges de manœuvre supplémentaires au niveau local, ça c'est clair. On avait une culture du partenariat du temps de l'ANPE qui était déjà forte, qui était déjà implantée, mais qui était (...) très dépendante de chaque directeur d'agence. Là, avec Pôle emploi 2015, j’aurais tendance à dire que le collègue qui ne veut pas y aller, il faut y aller quand même sur le partenariat, c'est-à-dire qu'on est nous aussi jugés là-dessus, dans le cadre du dialogue de performance, notre contrôle performance, ça fait partie de sujets qui sont évoqués. Donc il faut aller là-dessus. (...) Auparavant, on était beaucoup sur les thématiques de la co-traitance avec les missions locales, les maisons de l'emploi parce qu'on était membre de droit au conseil d'administration. Le RMI ${ }^{8}$, on participait au comité de suivi, mais après on s'impliquait plus

8. Revenu minimum d'insertion. 
ou moins. Avec le RSA, la loi a aussi défini le rôle de Pôle emploi et la mise en place de la fameuse concertation locale. Donc, tout ça, c'est cadré maintenant (...), et je trouve que Pôle emploi 2015 me donne plus de légitimité à aller chercher, à être créatif, essayer de trouver quelque chose de nouveau sur le territoire qui vienne en complément » (directeur agence Pôle emploi, Banlieue Idf).

Les indicateurs de performance semblent être à l'unisson de ces évolutions puisque désormais ce sont les placements et le délai de satisfaction des offres d'emploi qui sont évalués, ce qui incite Pôle emploi à solliciter ses partenaires, pour capter des candidats par exemple. Pôle emploi s’appuie aussi sur les autres intermédiaires pour relayer l'information sur son offre de services. Surtout, l'enjeu majeur pour Pôle emploi consiste à déléguer l'accompagnement des demandeurs d'emploi, qu'il n'a pas les moyens ou les compétences d'assurer (quand la taille des portefeuilles est au maximum ou pour tout le volet social de l'accompagnement), à des acteurs mieux ajustés aux échelles géographiques propres au public peu qualifié ; et en retour, à aider les structures à atteindre leurs objectifs en matière de retour à l'emploi, ce critère étant désormais celui qui prime dans les dialogues de gestion pour tous les intermédiaires :

«L'approche stratégique Pôle emploi 2015 dit qu'on doit nous, au niveau local, travailler sur une territorialisation de nos actions, chercher justement des dynamiques locales pour compléter notre offre de service. Mais moi derrière, aller voir des partenaires en disant : 'J'ai besoin que vous me suiviez des demandeurs d'emploi parce que moi je n'y arrive pas, je suis débordé"... au bout d'un moment, si moi je ne leur donne pas d'autres choses en échange, ils vont me dire : "OK, vous êtes bien gentils mais non, on arrête.” (...) Moi, j'ai intérêt à aider [les structures d'insertion par l'activité économique] à placer leur public, à les sortir sur les solutions d'emploi durables parce qu'en plus, eux, ça les aide à atteindre leurs objectifs, surtout avec la réforme de l'IAE, ça va être important. Notamment sur la partie modulable de l'aide, le taux de sortie va jouer dans le calcul, forcément. Donc ça c'est dans leur intérêt, mais moi c'est dans mon intérêt parce que plus ils en sortent, plus ils peuvent me prendre aussi des personnes en accompagnement IAE » (directeur Pôle emploi, territoire 2).

Ainsi, l'opérateur public de placement participe à la régulation du champ des intermédiaires en pilotant la répartition des demandeurs d'emploi. D’un point de vue opérationnel, ces relations bilatérales entre Pôle emploi et les autres intermédiaires prennent la forme :

- de partenariats institutionnels souvent qualifiés de « naturels », parce qu'ils sont rendus obligatoires par la définition légale du SPE. Il s'agit là de partenariats avec d'autres instances d'élaboration des politiques de l'emploi (Direccte, Conseil départemental, Région), avec des homologues 
opérationnels (mission locale, Cap emploi, structures RSA) ou bien encore avec l'insertion par l'activité économique (Pôle emploi participant au dialogue de gestion des structures IAE avec la Direccte);

- de partenariats de régulation qui consistent à organiser matériellement la circulation des demandeurs d'emploi vers les opérateurs privés de placement (OPP) ou les organismes de formation, selon le volume et le rythme déterminé au préalable par contrat ;

- de partenariats émergents - ou choisis - en fonction de l'offre et des besoins sur le bassin d'emploi. C'est sur ce segment que les directeurs d'agence sont libres d'être créatifs, de construire des partenariats avec des associations (Nos quartiers ont du talent, Solidarité nouvelle contre le chômage, divers organismes d'aide à la création d'entreprise), avec des homologues publics (maisons de l'emploi, structures municipales), des concurrents privés (ETT). C'est là que l'on observe également des différences d'un territoire à un autre, avec des partenaires très présents sur certains terrains et quasi absents sur d'autres. À titre d'exemple, sur le territoire Banlieue IdF, les directeurs de l'agence Pôle emploi et de la maison de l'emploi décrivent plusieurs actions en commun : l'organisation du forum des emplois de la ville, des opérations de recrutement, des animations d'ateliers collectifs.

Un autre exemple de relations bilatérales est donné par celles nouées par la mission locale de Banlieue IdF, dont l'enclavement au sein d'un quartier de la commune a déjà été souligné. Elle est néanmoins parvenue à tisser des liens avec des acteurs qui œuvrent justement à désenclaver la population du territoire : les échelons locaux des centres d'information et de documentation jeunesse, au sein desquels un salarié de la mission locale est détaché plusieurs jours par semaine pour une permanence. La mission locale parvient ainsi à toucher le public appartenant à l'autre moitié de la commune (coupée en deux de façon latente) tandis que le point information jeunesse bénéficie d'un intervenant susceptible de diffuser informations et conseils quant à l'insertion professionnelle. Les travailleurs sociaux et les éducateurs de rue constituent également des relais de poids pour la mission locale qui, par leur intermédiaire, est susceptible d'augmenter son audience auprès de jeunes qu'elle n'était pas encore parvenue à toucher. Le partage d'un même public et d'une même échelle d'intervention - en l'occurrence ici la commune voire le quartier - produit une convergence des intérêts qui favorise le bon fonctionnement des relations bilatérales. Se pose ainsi la question de l'échelle territoriale à laquelle peut se produire cette coordination des différents intermédiaires. On observe sur cette question des différences entre les deux terrains. 


\section{II.2. Coordination des échelles d'intervention et (dé)stabilisation des dynamiques locales}

Si, comme on l'a vu précédemment, les échelles d'intervention varient selon les intermédiaires, on peut néanmoins distinguer, sur chacun des territoires enquêtés, une échelle privilégiée au niveau de laquelle se structurent les politiques locales de l'emploi et se coordonnent les différents acteurs, échelle méso pour Rurbain platlog et micro pour Banlieue IdF.

L'échelle de coordination privilégiée sur Rurbain platlog découle très largement des spécificités productives et démographiques du territoire. D’abord, l'absence de centre urbain dominant réduit les velléités impérialistes d'une équipe municipale ou d'une agence de Pôle emploi. Ensuite, les employeurs y délèguent aux intermédiaires publics et privés le travail de mobilisation d'une force de travail que leurs politiques RH participent très largement à épuiser. Sur Rurbain platlog, se trouve en effet un important gisement d'emplois peu qualifiés, situé sur une zone d'activité logistique accueillant peu ou prou 14000 travailleurs. Faiblement qualifiée, marquée par des conditions de travail physiquement épuisantes et une absence de politiques des entreprises pour y remédier, cette activité d'entreposage fonctionne en s'appuyant sur un important turn-over. L'intérim d'abord et les intermédiaires publics ou associatifs du marché du travail sont alors très largement mobilisés pour élargir continuellement la prospection de travailleurs et de travailleuses potentielles. Avec le jeu des primes de précarité, certaines activités de manutention peu qualifiées permettent de dégager des revenus supérieurs à la moyenne de ce type d'emploi. Cette situation a différents effets sur le fonctionnement du marché du travail local. D’une part, l'importance du turn-over conduit les employeurs du secteur à se tourner vers des intermédiaires de l'emploi habituellement occupés à placer des travailleurs plus qualifiés (les entreprises de travail temporaire notamment). D’autre part, les salaires pratiqués permettent à des travailleurs non qualifiés de l'ensemble du bassin d'emploi d'assumer des trajets longs, du moins lorsqu'ils sont véhiculés. L'activité de la plupart des intermédiaires de la zone est orientée par cette activité : les très nombreuses ETT, on l'a dit, sont largement mobilisées. L'agence Pôle emploi la plus proche de la plateforme consacre elle aussi une part importante de ses ressources humaines à saisir et diffuser les offres du secteur logistique et à rechercher des candidats pour les agences d'intérim. Cette situation oriente également l'activité de la seconde agence du territoire même si, plus éloignée des entrepôts, elle ne participe pas à la collecte des offres. Ses inscrits sont en effet fortement aimantés par les emplois de la plateforme, du moins lorsqu'ils ont les moyens de s'y déplacer. Cette conjonction de facteurs légitime les acteurs publics auprès des employeurs et favorise la collaboration des intermédiaires puisqu'ils sont relativement atomisés. L'animateur 
territorial rattaché à la Direccte se trouve dès lors être l'acteur le mieux doté pour gérer la circulation de l'information sur l'évolution des implantations productives et tenter de coordonner la répartition du travail entre les différents intermédiaires locaux. Aussi, sur ce premier territoire, on observe une certaine convergence vers une échelle dominante d'intervention des intermédiaires - le niveau du bassin d'emploi - en concordance avec les qualifications et les aires de mobilité de la plupart des « chercheurs d'emploi ${ }^{9}$ » ainsi que les opportunités d'emploi locales. Ces habitudes de coordination se maintiennent dans d'autres types de projets de développement local ayant un volet emploi, comme les relocalisations industrielles, l'implantation de villages de vacances ou de magasins d'usine. La légitimité acquise par l'animateur territorial de la Direccte se fait alors pleinement sentir.

Cette concordance des échelles entre opportunités et aires de recherche d'emploi ne s'observe pas sur le second territoire. La désynchronisation provient du décalage entre le périmètre d'action de la majorité des intermédiaires - qui interviennent à une échelle très localisée du fait des difficultés du public en présence - et les opportunités d'emploi locales très qualifiées, qui recrutent sur un bassin d'emploi macro. Cela complique la coordination des différents intermédiaires. On ne trouve d'ailleurs pas, sur ce territoire, d'instance « chef d'orchestre » unanimement reconnue par tous. Au moment de notre enquête, on pouvait néanmoins relever une certaine convergence d'échelles entre, d'une part, l'accompagnement de proximité proposé par les structures municipales et d'autres acteurs locaux avec lesquels étaient noués des partenariats et, d'autre part, la politique de développement économique local menée par les mêmes structures. La maison de l'emploi du territoire Banlieue IdF mettait en effet en œuvre un accompagnement à l'emploi de publics défavorisés jeunes, au travers de la mission locale, et moins jeunes via le pôle insertion. Ce pôle insertion travaillait au plus près des quartiers et en partenariat avec les structures sociales du territoire qui prennent en charge ces publics au quotidien (centre d'action sociale, éducateurs de rue...). L'accompagnement des publics s'effectuait à une échelle très localisée voire ultra-locale, qui reposait néanmoins sur un débouché important : les offres d'emploi relatives aux clauses d'insertion dans le cadre des projets de rénovation urbaine (Anru). Celles-ci étaient gérées par une « facilitatrice » elle aussi localisée au sein de la maison de l'emploi (et anciennement employée au pôle insertion). Cette proximité semblait permettre une progressive synchronisation des échelles d'intervention - le volume de personnes suivies était réduit mais avec une montée en qualification. Les évolutions ayant ensuite affecté les maisons de l'emploi ont remis en cause cette

9. Nous parlons ici de chercheurs d'emploi dans la mesure où c'est sous cette forme que le public est modélisé par les différentes institutions vouées à les prendre en charge. Mais il faut bien garder à l'esprit que ces personnes ne peuvent se réduire à cette composante de leur existence. Ce sont les politiques d'activation qui ont contribué à ce glissement. 
dynamique. L'interdiction qui leur est faite d'accompagner du public a conduit à la fermeture du pôle insertion et à la délocalisation de la mission locale. Ces orientations changeantes dues à des réformes dites de « simplification » sont difficiles à gérer pour les acteurs de ces structures, elles désorganisent fortement leur travail. En outre, le progressif abandon des fonctions d'accueil du public et d'accompagnement laisse dans la nature des demandeurs d'emploi qui semblent ne pas pouvoir être pris en charge ailleurs car peu adaptés aux outils de recherche «a-territoriaux » ou « qualifiés » largement mis en œuvre par Pôle emploi (Fretel et al., 2016).

Les deux terrains témoignent de la variété des arrangements locaux trouvés par les intermédiaires du marché du travail pour articuler les politiques de l'emploi aux spécificités économiques locales. La coordination des structures au niveau local ne passe pas toujours par le leadership d'un des acteurs jouant le rôle de chef de file mais aussi par de multiples instances de concertation et commissions, conventions bilatérales ou multilatérales. On observe des convergences des échelles d'intervention des intermédiaires vers un niveau cohérent avec le tissu économique local (Rurbain platlog) ou pour répartir des « chercheurs d'emploi » entre des intermédiaires intervenant tous à une échelle micro (Banlieue IdF). Néanmoins, ces espaces de discussion ne sont pas exempts de jeux de positionnement et de conflits entre les participants ou de suspicion des uns et des autres quant aux modalités de répartition des différentes ressources allouées (débouchés d'accompagnement, offres d'emploi ou places en formation). Les parties prenantes sont en concurrence pour ces mêmes ressources et peuvent s'estimer lésées. La concurrence entre les acteurs locaux des politiques de l'emploi concerne aussi la question du pouvoir et du mandat de pilotage. Elle porte enfin sur les ressources budgétaires délivrées par les conseils départementaux, la Direccte, les mairies ou les régions et disponibles au niveau local. Cette concurrence latente des intermédiaires locaux du marché du travail ne peut être rabattue à une tendance à l'impérialisme qui les caractériserait tous. Une grande partie de ces tensions trouve sa source dans le mouvement récurrent des réformes, qui oblige les acteurs locaux à redéfinir constamment leurs pratiques et relations mutuelles en vertu d'une lecture toujours spécifique des transformations en cours et des moyens dont ils disposent. Ce constat est particulièrement bien illustré par la concurrence institutionnelle que se sont livrée l'ANPE, les Assedic et les maisons de l'emploi à la suite de la loi de cohésion sociale du 18 janvier 2005. Nos observations du terrain montrent qu'une répartition apaisée des pouvoirs et qu'une division stabilisée du travail opérationnel trouve toutes les peines à se mettre en place dans ce contexte qui favorise la collaboration compétitive ou « coopétition » (Bruno et al., 2006). S’il apparaît illusoire de vouloir mettre fin à de telles luttes de pouvoir institutionnelles par décret, 
il apparaît également que la succession sans fin des décrets favorise l'instabilité du système d'acteurs.

\section{Conclusion}

Quand on se prend à regarder ce qu'il se passe à distance de l'État central, on peut alors distinguer différentes échelles d'intervention pour les intermédiaires du marché du travail : macro, méso et micro. Chacun des acteurs étudiés relève d'une échelle d'intervention qui lui est propre, c'està-dire agit sur un espace circonscrit sur lequel il est légitime à intervenir. L'étendue plus ou moins grande du périmètre d'intervention de chaque structure dépend de nombreux facteurs mais notamment de la densité de son public cible et du périmètre de recherche d'emploi et de mobilité de ce public. Les échelles d'intervention paraissent ainsi souvent bien ajustées aux problématiques des demandeurs d'emploi suivis et correspondent à différentes visions du territoire, avec notamment des conceptions opposées pour Pôle emploi et les acteurs municipaux. D’un côté, Pôle emploi constitue un organisme multi-échelle mais c'est un acteur en partie a-territorialisé - en tous cas les critères territoriaux ne sont pas un critère pertinent pour favoriser tel ou tel demandeur d'emploi -, qui propose pour une bonne part une offre de service globale, pensée pour une intermédiation à l'échelle du bassin d'emploi mais déconnectée des enjeux de proximité des besoins locaux. Enfin, il n'a pas la main sur la question essentielle du développement économique local. De l'autre, pour les collectivités locales, le territoire n'est pas qu'une division administrative. Elles en ont une bonne connaissance, sont redevables vis-à-vis de ce territoire, c'est un critère pertinent et même à défendre pour elles. Elles s'appuient sur un réseau de partenaires de proximité pour s'occuper des plus fragiles et ont en partie la main sur les questions de développement économique. A contrario, elles n'ont pas l'expertise, les outils et le vivier de demandeurs d'emploi de Pôle emploi.

D'où l'émergence de partenariats entre ces deux acteurs majeurs au niveau local et les autres intermédiaires, d'instances de coordination pour synchroniser leurs différentes échelles d'intervention et articuler l'accompagnement des demandeurs d'emploi et le développement économique local à des échelles concordantes. Si de multiples relations bilatérales et instances de coordination se déploient sur le terrain, les atermoiements des politiques nationales, entre décentralisation et recentralisation créent des situations d'incertitude pour les acteurs locaux et sont sources de tensions et de concurrence pour le leadership. Si les deux monographies que nous avons menées ne peuvent permettre une montée en généralité non contrôlée, force est de constater que ces questions de coordination peuvent se 
régler différemment selon les territoires, en fonction de leur histoire, des rapports de force et personnalités en présence. Nous avons surtout souligné l'importance de prendre en compte le contexte économique local et la plus ou moins grande (in)adéquation d'échelle entre qualification, aire de mobilité des « chercheurs d'emploi » d'un côté et le tissu économique local d'un autre côté.

Un tel constat ne plaide pas forcément pour donner l'ensemble du pouvoir aux échelons locaux mais plutôt pour l'instauration de dispositifs de coordination susceptibles de mettre en convergence les différences initiatives - y compris celles dont l'État déconcentré est à l'origine - et les échelles d'intervention adéquates concernant le développement économique local et l'accompagnement des demandeurs d'emploi. La question de savoir comment l'État peut agir pour favoriser cette combinaison des différentes échelles d'intervention et l'ajuster aux spécificités de chaque territoire reste plus que jamais d'actualité. 


\section{Références bibliographiques}

Balzani B., Béraud M., Boulayoune A., Divay S., Eydoux A., Gouzien A. (2008), L'accompagnement vers l'emploi. Acteurs, pratiques, dynamiques, Rapport final pour la Dares, septembre, https://halshs.archives-ouvertes.fr/ halshs-00377437/document.

Baron C., Bureau M.-C., Leymarie C., Nivolle P. (1995), « L'action des intermédiaires : animation du marché local du travail, mobilisation de la main d'oeuvre, médiation locale », Cahier du Centre d'études de l'emploi, n 34 , p. 213-233.

Bartoli J.-R., Mériaux O. (2011), « Les politiques de l'emploi au risque de la territorialisation concurrentielle », in Pasquier R., Guigner S., Cole A. (dir.), Dictionnaire des politiques territoriales, Paris, Presses de Sciences Po.

Bel M. (2007), « Formation et territoire : des approches renouvelées », Formation Emploi, n 97, p. 67-80, https://www.cairn.info/revue-formation-emploi2007-1-page-67.htm.

Berhuet S. (2012), « L'inflexion des Maisons de l'Emploi en faveur du développement local des territoires ", in Bureau M.-C., Sainsaulieu I. (dir.), Reconfigurations de l'État social en pratique, Villeneuve d'Ascq, Presses universitaires du Septentrion, p. 233-248, https://hal.archives-ouvertes.fr/hal-00697402.

Berhuet S., Tuchszirer C. (2015), « Les maisons de l'emploi ou l'introuvable politique territoriale de l'emploi », Connaissance de l'emploi, n 118, http://bit. ly/30OWlo4.s.

Berthet T. (2008), « Usages de la proximité dans l'action publique en matière d'emploi-formation », in Baron C., Bouquet B., Nivolle P. (dir.), Les territoires de l'emploi et de l'insertion, Paris, L'Harmattan, p. 85-104.

Berthet T. (2010), " Externalisation et gouvernance territoriales des politiques actives de l'emploi », Revue française de Socio-Économie, n 6, p. 131-148. https://doi.org/10.3917/rfse.006.0131.

Berthet T., Cuntigh P., Guitton C., Mazel O. (2002a), « 1982-2002 : la territorialisation progressive des politiques de l'emploi », Premières Synthèses, $n^{\circ} 24.2$, juin, http://bit.ly/2YW01rH.

Berthet T., Cuntigh P., Guitton C. (2002b), « Politique d'emploi et territoires », Cereq Bref, $n^{\circ} 182$, janvier, http://www.cereq.fr/cereq/b182.pdf.

Bezes P., Le Lidec P. (2010), « L'hybridation du modèle territorial français : RGPP et réorganisations de l'État territorial ", Revue française d'administration publique, vol. 136, n 4, p. 919-942, https://doi.org/10.3917/rfap.136.0919.

Borel B., Ferras B., Le Coz G., Ville C. (2013), «Évaluation de la politique territoriale de l'emploi. Cartographie, bilan et recommandations (MAP) ", Rapport $n^{\circ}$ RM2013-164P, Igas, mai, http://www.igas.gouv.fr/IMG/pdf/ RM2013-164P_definitif_.pdf.

Bruno I., Jacquot S., Mandin L. (2006), « Europeanization through its instrumentation: benchmarking, mainstreaming and the open method of co-ordination... toolbox or Pandora's box? ", Journal of European Public Policy, vol. 13, $n^{\circ} 4$, p. 519-536, https://doi.org/10.1080/13501760600693895.

Canceill G., Pignoni M. T. (2000), «Introduction au dossier "Acteurs locaux de l'emploi" », Travail et Emploi, n81, p. 5-7, http://bit.ly/2WkdyfO. 
Castel R. (1995), Les métamorphoses de la question sociale : une chronique du salariat, Paris, Fayard.

Crague G. (2018), « Le couplage développement économique/emploi dans les territoires. L'expérience singulière des grands groupes industrialisateurs », Communication au Cnefop, Inter-commission Évaluation et Territoires, séance du 14 juin.

de Barros F. (2001), "Secours aux chômeurs et assistances durant l'entre-deuxguerres. Étatisation des dispositifs et structuration des espaces politiques locaux », Politix, vol. 14, n53, p. 117-144, https://doi.org/10.3406/ polix.2001.1140.

Doan Q. C. (2015), « Mobiliser les territoires : la preuve par le SPE », Synthèses, $n^{\circ}$ 6, DGEFP, https://www.profildinfo.fr/doc_num.php?explnum_id=10094.

Duran P., Thoenig J.-C. (1996), « L'État et la gestion publique territoriale », Revue française de science politique, vol. 46, $\mathrm{n}^{\circ} 4$, p. 580-623, https://doi. org/10.3406/rfsp.1996.395082.

Epstein R. (2015), « La gouvernance territoriale : une affaire d'État. La dimension verticale de la construction de l'action collective dans les territoires ", L'Année sociologique, vol. 66, $\mathrm{n}^{\circ}$ 2, p. 457-482, https://doi.org/10.3917/ anso.152.0457.

Fondeur Y. (2016), « Dynamiques écologiques du marché du travail en ligne autour de la circulation des offres d'emploi ", in "Diversité et dynamiques des intermédiaires du marché du travail ", Études et Recherches, vol. 7, Pôle emploi et Rapport de recherche, n 100, CEE, p. 165-212, http://bit. ly/2ljyLfR.

Fretel A., Pillon J.-M., Remillon D., Tuchszirer C., Vivés C., Fondeur Y. (2016), « Dynamiques territorialisées du champ de l'intermédiation ", in Diversité et dynamiques des intermédiaires du marché du travail, Études et Recherches, vol. 7, Pôle emploi et Rapport de recherche, n 100, CEE, p. 5-164, http:// bit.ly/2Km5ZOx.

Legay A., Monchatre S. (2000), « L'ANPE à l'épreuve du local », Travail et Emploi, $n^{\circ} 81$, p. 41-51, http://bit.ly/2wANo9n.

Marchal E., Bureau M.-C. (2009), « Incertitudes et médiations au cœur du marché du travail », Revue française de sociologie, vol. $50, n^{\circ} 3$, p. 573-598, https:// doi.org/10.3917/rfs.503.0573.

Marcou G. (2012), « Changements et permanences dans le système français d'administration territoriale ", Revue française d'administration publique, vol. 141, n 1, p. 5-17, https://doi.org/10.3917/rfap.141.0005

Poupeau F.-M. (2013), « L'émergence d'un État régional pilote : La recomposition des jeux administratifs autour du ministère de l'Écologie et du Développement durable dans une région française ", Gouvernement et action publique, vol. 2, n² 2, p. 249-277, https://doi.org/10.3917/gap.132.0249.

Simha J. (2013), « La confrontation de deux instruments d'action publique : territorialiser la formation professionnelle », Économies et Sociétés, $n^{\circ} 35$, p. 759785, https://socioecotravail.hypotheses.org/files/2015/09/35-5-Simha2.pdf. 